\title{
Article \\ Determination of the Most Interconnected Sections of Main Gas Pipelines Using the Maximum Clique Method
}

\author{
Sergey Vorobev *, Anton Kolosnitsyn (D) and Ilya Minarchenko \\ Melentiev Energy Systems Institute SB RAS, 664033 Irkutsk, Russia; kolosnitsyn@isem.irk.ru (A.K.); \\ minar@isem.irk.ru (I.M.) \\ * Correspondence: seregavorobev@isem.irk.ru
}

check for updates

Citation: Vorobev, S.; Kolosnitsyn, A.; Minarchenko, I. Determination of the Most Interconnected Sections of Main Gas Pipelines Using the Maximum Clique Method. Energies 2022, 15, 501. https://doi.org/10.3390/en15020501

Academic Editor: Artur

Kierzkowski

Received: 7 December 2021

Accepted: 6 January 2022

Published: 11 January 2022

Publisher's Note: MDPI stays neutral with regard to jurisdictional claims in published maps and institutional affiliations.

Copyright: (C) 2022 by the authors. Licensee MDPI, Basel, Switzerland. This article is an open access article distributed under the terms and conditions of the Creative Commons Attribution (CC BY) license (https:// creativecommons.org/licenses/by/ $4.0 /)$.

\begin{abstract}
This article is devoted to the definition of the most important combinations of objects in critical network infrastructures. This study was carried out using the example of the Russian gas transmission network. Since natural gas is widely used in the energy sector, the gas transmission network can be exposed to terrorist threats, and the actions of intruders can be directed at both gas fields and gas pipelines. A defender-attacker model was proposed to simulate attacks. In this model, the defender solves the maximum flow problem to satisfy the needs of gas consumers. By excluding gas pipelines, the attacker tries to minimize the maximum flow in the gas transmission network. Russian and European gas transmission networks are territorially very extensive and have a significant number of mutual intersections and redundant pipelines. Therefore, one of the approaches to inflicting maximum damage on the system is modeled as an attack on a clique. A clique in this study is several interconnected objects. The article presents the list of the most interconnected sections of main gas pipelines, the failure of which can cause the greatest damage to the system in the form of a gas shortage among consumers. Conclusions were drawn about the applicability of the maximum clique method for identifying the most important objects in network critical infrastructures.
\end{abstract}

Keywords: gas transmission network; attacker-defender model; maximum clique problem; energy security

\section{Introduction}

The transport schemes of energy systems over time undergo significant changes, both in the connections between the nodes and in the production capabilities of these connections and nodes, and in the number of these nodes themselves and the connections between them. In the gas industry, with an increase in the degree of development of old long-standing fields, it becomes necessary to enter new gas-bearing provinces with a corresponding development of the gas transportation infrastructure. In turn, the transport infrastructure, which is directly related to old sources of production that are decreasing in their productivity, is used less and less over time, which is caused by a natural decrease in load. Thus, the gas transmission network is constantly changing over time, both in terms of its configuration and the loading of its elements.

At the same time, a complex gas transmission network contains a significant number of facilities, the performance of which critically affects the overall performance of the gas transmission network and, accordingly, its ability to reliably provide an uninterrupted gas supply to consumers. The most large-scale accidents in power systems occur as a result of the malfunction of various critical objects of these systems [1,2]. One of the results of such accidents is great damage to consumers, which is expressed in a large shortage of fuel and energy resources. Therefore, the identification of critical objects and their combinations in power systems in order to develop measures aimed at reducing the significance of these objects is an urgent task. 
Among the studies devoted to various aspects of the vulnerability of critical infrastructures and energy systems, the closest to the problems considered in this article are the following works.

The identification of critical components in a pipeline network is an essential part of vulnerability analysis. The authors proposed a new risk vulnerability analysis method that combines both the characteristics of risk assessment methods and vulnerability analysis methods [3]. Thus, in this study, the vulnerability of critical components of a pipeline network is determined from three points of view: the operational state of the pipeline, transmission performance, and network performance.

The characteristics of fluctuations in gas demand and the impact of user importance are usually ignored in previous studies of gas supply reliability. To overcome these shortcomings, the study [4] proposed an integrated demand analysis method for assessing the reliability of gas supply to a large-scale and complex natural gas pipeline network. The method consists of creating an indicator system, analyzing demand, assessing the probability of unit failure, and calculating gas supplies. The gas flow calculation takes into account the influence of the relationship of user importance, hydraulic and pressure limits, and unit failure. In addition, the real natural gas pipeline network located in China is being used to validate the feasibility of this method. Based on the results of assessing the reliability of gas supply, the weakest nodes and key links of the gas pipeline network were identified, and proposals were made to improve the reliability of gas supply. Finally, the importance of demand in the reliability of gas supply was worked out and substantiated.

The researchers also considered the problems of the vulnerability of critical energy infrastructures to terrorist attacks on them, and also proposed a methodology for analyzing risks for systems of interdependent critical infrastructures under various extreme weather events $[5,6]$.

A multidimensional framework for the analysis of critical infrastructures in terms of supply services, topology, and manageability was proposed in [7]. The framework allows researchers to define the role of infrastructure elements and quantify the consequences of multiple failures of each specific element. As an example, a reference network is considered, representing a real gas transmission network in several countries of the European Union.

The dangers that can cause direct local damage or disruption of the system, and which are defined as spatially localized attacks, are listed in [8]. Their impact on critical infrastructure is modeled as the failure of system components that exist in a localized area, while those outside this area continue to operate. The study analyzed the worst case and proposed a mathematical basis to support the optimization of the resilience of interdependent critical infrastructures in the worst spatially localized attacks. For illustrative purposes, the article mainly discussed two types of strategies for increasing the resiliency of critical infrastructures, including protecting weak components and creating new components to increase reliability. The problem was mathematically formulated as a three-level model of defender-attacker-defender, which was solved using the decomposition algorithm.

Another article analyzed scientific work on the sustainability of supplies of biomass, water, electricity, and natural gas [9]. The results showed that existing research favors fewer systemic strategies when optimizing resilience. The review concluded that optimization is a useful tool for continually achieving sustainability in the supply chain associated with the production, storage, and transportation of resources.

A comprehensive risk assessment scheme for predicting failures and consequences of failures of offshore oil pipelines is presented in [10].

The authors proposed a method to assess the risk in terms of two points: the cumulative probability of failure of the pipeline and its leak detection system, as well as the magnitude of the consequences of such a failure. The consequences are financial losses associated with environmental damage, oil spill response, and production losses. Ultimately, this analysis provides an estimate of the risk in monetary terms and helps to determine whether the assessed risk exceeds a predetermined target risk. 
In [11], it was mentioned that oil and gas pipelines are more vulnerable to natural and deliberate threats due to their large territorial extent. This study presents and discusses potential needs in research fields such as pipeline safety, environmental sustainability, and pipeline system sustainability to improve the sustainability of oil and gas pipelines. Research on risk assessment based on Bayesian networks may expand in the future. In addition, more studies and recommendations are expected on pipeline safety, sustainability, and environmental impact to better protect pipelines.

In the studies reviewed, single critical elements of infrastructure systems are identified. Situations with the failure of several elements of the system at the same time are not considered.

The following studies are devoted to the identification of critical objects in the gas industry. A list of objects of the Unified Gas Supply System of Russia, the disruption of which will lead to a relative deficit of daily gas supplies to consumers in the amount of $5 \%$ or more of the total needs in the industry, was determined [12]. Studies to determine the combinations of objects, the simultaneous failure of which can lead to more than a $5 \%$ shortage of daily gas supplies through the system were carried out $[13,14]$. The study [15] was devoted to the identification of critical objects of the electric power industry in Russia. After analyzing the results of previous studies, taking into account the work carried out around the world, a methodology for determining the critical objects of energy systems was developed. This methodology was tested on the example of the Russian gas industry $[16,17]$.

In reviewed works, the determination of critical objects and their combinations were carried out using the method of enumeration. Multi-iteration studies were carried out in which all elements and pairs of elements were switched off in turn in the computational network. Thus, we determined those elements of the network and their combinations where their failure would lead to the greatest gas deficit in the system. Due to restrictions on the amount of analyzed information, situations with a one-time disconnection of three or more network elements were not considered. Nevertheless, due to the specifics of the functioning of the gas transmission network, such emergency situations of a cascade nature are possible. Therefore, for greater completeness and comprehensive consideration of various factors in the study of critical objects in this work, the authors use the method of determining cliques to find the maximum number of interconnected main gas pipelines where their failure would cause significant damage to the system in terms of reducing gas supplies to consumers.

The problem of determining the maximum clique is formulated within the framework of the problem of modeling attacks on infrastructure systems. Here, the attacking side and the defending side are connected in a single mathematical formulation. Such models are based on the class of Stackelberg games [18] with constraints in networks. In them, two characters-a leader and a follower-pursue opposing interests. The goal of the attacker is to inflict maximum damage on the defender under given resource constraints by reducing the bandwidth or increasing transport costs on certain arcs, or completely removing nodes or arcs from the computational network. The defender solves their optimization problem on the network changed by the attacker. A detailed description of mathematical models of this type of problem can be found in [19]. We also note that the mathematical description of such problems can be reduced to multilevel optimization models of the attacker-defender, defender-attacker, and defender-attacker-defender types, which have found wide application in scientific research in modeling threats and response measures at various objects of critical infrastructures. In fact, such models allow for determining the optimal plan for implementing protective actions with a limited budget. A comprehensive analysis of different types of defender-attacker-defender models is presented in [20]. Theoretical and mathematical foundations of interdiction games and their applications are given in [21]. In this work, we were primarily interested in the reliable functioning of the gas transmission system from the standpoint of its protection from terrorist attacks. For this purpose, a model of the attacker-defender type was considered in which the solution of the attacker's 
problem was based on the problem of finding the maximum clique. The protector's job was to maximize the flow of gas through the network. Such a formulation will make it possible to identify those critical network objects and their combinations where their failure leads to the greatest decrease in the network bandwidth as a whole.

\section{Materials and Methods}

\subsection{Threat Modeling at Critical Infrastructures}

Several studies proposed the approach that combines an attacker's and a defender's decisions in optimization-based models for the purpose of protecting critical infrastructures. Such models can be represented as a three-stage sequential game and commonly take the form

$$
\text { (DAD) } \min _{w \in W} \max _{x \in X(w)} \min _{y \in Y(w, x)} f(y) \text {. }
$$

In the first stage, the defender chooses the plan of necessary infrastructure investments $w \in W$. In the second stage, the attacker forms their attack plan $x \in X(w)$ by considering the defender's actions. In the third stage, the defender collects all information about the investments and corresponding attack plan and then chooses the plan of activities $y \in Y(w, x)$ that minimizes the operating cost defined by means of the function $f(y)$.

The resulting formulation is called the DAD model. Solving an internal problem, the defender acts as an operator and finds such a set of actions that allows for minimizing the costs of operating the infrastructure system. The designation $y \in Y(w, x)$ indicates that the operator's actions depend on the plan $x$ of the attacking side, as well as on the preliminary actions to protect the system. The operator's costs can be expressed not only in a monetary sense but also, for example, by a certain output of a product or the flow of electricity through the network.

For operating infrastructure systems, as a rule, some common mathematical models are applied to describe the interactions between the main objects of these systems. A flow and supply model is often used for describing a power infrastructure system [22-24]. As for the components of a water distribution system, the standard hydraulic model should be used [25]. The standard traffic flow model is commonly applied to account for the cost of road network components [26].

The maximization operation of the DAD model represents the actions of the attacker. The attacker takes into account the preparatory actions of the defender and chooses an attack plan $x \in X(w)$. The attacker tends to maximize the damage of the operator by maximizing the costs of operating the system.

Finally, external minimization in a DAD model represents the actions of a defender who chooses a defensive investment plan $w \in W$. This plan can include active defenses, hardening the infrastructure against attacks or creating new infrastructure that is less vulnerable to attacks. The defender's plan $w \in W$ takes into account the limited resources, which need to be allocated in the best way to maximize the attack resistance of the infrastructure system. In this case, the solution of the DAD-type problem determines such system components that should be protected to minimize the destructive actions of the attacker.

Note that two-stage problems of the attacker-defender and defender-attacker types can also be considered. In the model of the first type, a part of the external minimization is omitted that corresponds to the actions of the defender to strengthen the components of the model. In this case, the problem is formulated as follows:

$$
\max _{x \in X} \min _{y \in Y(x)} f(y)
$$

The second type of problem arises in cases when the internal problem of network parameter optimization is lacking or is solved in a trivial way; then, the attacker-defender model can be obtained in the following form:

$$
\min _{w \in W} \max _{x \in X(w)} g(w, x),
$$


where $w$ is a vector of the defender's choices, $W$ is its feasible set, and $g$ is the objective function that represents the damage inflicted to the network. Such a model occurs, for example, in territorial boundary patrol problems described in [20].

Generally, the DAD models in the form of Equation (1) are difficult to solve, given that it is often impossible to reduce it to mixed-integer programming problem and complex decomposition techniques are to be applied. An example that involves applying Bender's decomposition to solve a DAD-type problem is given in [27].

\subsection{Mathematical Model of the Gas Transmission System}

The gas transmission system of Russia is currently a very extensive gas transportation network. It is geographically distributed over many thousands of kilometers, with numerous intersections of major gas pipelines. The calculation scheme used in this study fully reflects all the technological features of a real gas transmission network and includes 388 nodes, among which, there are 96 consumers corresponding to the regions of gas consumption and individual large production consumers, 33 gas production fields, 29 underground gas storage facilities, and 230 nodal compressor stations.

Note that the nodes corresponding to underground gas storages can be involved in the system both as consumers of gas (if it is necessary to store it) and as producers (if the accumulated reserves are necessary to meet consumer demand).

The connection between the nodes is established by means of 755 gas pipeline lines. From the official statistical information [28-30], the following initial data for modeling were taken: volumes of gas production at the fields, the required volumes of demand at the nodes of consumption, and the throughput capacity of gas pipelines.

To solve the problem of supplying consumers with the required volumes of natural gas, the maximum flow problem was used. This formulation required the following notation. I is the set of nodes of the model; $I_{p} \in I$ is the set of nodes of gas deposits; $I_{s} \in I$ is the set of consumer nodes; $I_{0} \in I$ is the set of branch nodes; $S$ is the set of arcs (pipelines) of the model; $f$ is the total network flow; $U$ is the adjacency $n \times n$ matrix with elements

$$
u_{i j}=\left\{\begin{array}{c}
1, \text { node } i \text { is connected to node } j, \\
0, \text { node } i \text { is not connected to node } j ;
\end{array}\right.
$$

$x_{i j}$ is the value of the flow outgoing from the node $i$ and entering the node $j$, where the corresponding arc has the designation $(i, j) ; d_{i j}$ is the flow capacity of the arc $(i, j) ; b_{i}, i \in I_{p}$, is the gas production; $b_{i}, i \in I_{S}$, is the gas consumption; $i_{0}$ is the number of the fictitious nodes representing the cumulative source;

$$
b_{i 0}=\sum_{i \in I_{p}} b_{i} ;
$$

$i_{S}$ is the number of fictitious nodes that represent the total drain;

$$
b_{i s}=\sum_{i \in I_{c}} b_{i} .
$$

The problem of finding the maximum flow through the network has the following meaningful interpretation: it is necessary to find the maximum possible total volume of gas that can be passed through the network with the given characteristics of connections between the nodes, taking into account the established capacities of the lines, the available production volumes, and the given volume of consumption. Solving the problem of finding the maximum flow through the network makes it possible to determine whether the system is capable of providing consumers with the required volume of gas that is delivered to them through the gas pipeline network. This formulation does not take into account the gas consumption for the needs of the gas transmission network itself. To account for this flow rate in the current study, the gas flow rate for the entire system was increased by $10 \%$. 
This indicator was obtained as a result of previously conducted numerous technical and economic studies of the functioning of the gas transmission systems [31].

For the correct operation of the maximum flow search algorithm, it was necessary to perform the following additional operations:

1. Add to set $I$ two fictitious nodes with numbers $i_{o}$ and $i_{s}$, corresponding to the total source and total drain. Connect all production nodes to a dummy node $i_{0}$ :

$$
u_{i j}=1, i=i_{0}, j \in I_{p} .
$$

2. The line capacity $d_{i j}, i=i_{0}, j \in I_{p}$ is set equal to the volume of gas production at the nodes $j \in I_{p}$. Connect all consumer nodes to a fictitious node $i_{s}$ :

$$
u_{i j}=1, i \in I_{c}, j=i_{s} .
$$

3. The line capacity $d_{i j}, j=i_{s}, i \in I_{c}$ is set equal to the volume of consumption in the nodes $i \in I_{c}$. Establish the equivalence of variables:

$$
x_{i j}=x_{j i}, i, j \neq\left\{i_{O}, i_{S}\right\} .
$$

4. Set the capacity of the lines, taking into account the symmetry of the adjacency matrix $U$ :

$$
0 \leq x_{i j} \leq d_{i j}, 0 \leq x_{j i} \leq d_{i j}, i \in I, j \in I .
$$

5. Introduce matrix $\bar{U}$ with elements $\bar{u}_{i j}$, which are defined as follows:

$$
\bar{u}_{i j}=\left\{\begin{array}{c}
u_{i j}, \text { if } i<j, \\
-u_{i j}, \text { if } i>j .
\end{array}\right.
$$

Then the statement of the maximum flow problem has the following form:

$$
\begin{gathered}
\max f, \\
\sum_{j \in I} \bar{u}_{i j} x_{i j}=\left\{\begin{array}{c}
-f, i=i_{O}, \\
0, i \notin\left\{i_{O}, i_{S}\right\}, i \in I, \\
f, i=i_{S},
\end{array}\right. \\
x_{i j}=x_{j i}, \quad i, j \in I \backslash\left\{i_{O}, i_{S}\right\}, \\
0 \leq x_{i j} \leq d_{i j}, \quad i \in I, j \in I .
\end{gathered}
$$

Information on the ability of the gas transmission system to cover the load from consumers became available to us after solving Problem (2). Bottlenecks can also be identified, which are sections of the gas pipeline with the maximum load and sections with a large margin of throughput. These data can contribute to making adjustments to the characteristics of the gas transmission system in the direction of increasing or decreasing the throughput of certain gas pipelines, taking into account the load on the part of consumers.

\subsection{Statement of the Maximum Clique Problem}

\subsubsection{Basic Problem Statement}

For the gas transmission system in Russia, we proposed to identify the most vulnerable attack targets as a combination of nodes in which each node is connected to each other node in the combination. In other words, the problem is to find a clique of the maximum size [32]. For clarification, we use the terminology from graph theory.

The gas transportation system was represented using a directed graph. The nodes of such a graph are fields, gas-consuming regions, underground gas storage reservoirs, and nodal compressor stations. The edges of this graph are the gas pipelines. The main goal of the attacker is to create the greatest damage to the gas transmission system. In this study, 
damage meant a decrease in the maximum gas flow through the gas pipeline network by disabling the nodal or linear objects of the system. Naturally, fictitious nodes $i_{o}$ and $i_{s}$ are not considered when planning an attack and searching for a clique.

We assumed that the attacker solves the problem of finding the maximum clique in order to disable the largest number of interconnected objects of the gas transmission system. Here is a list of the basic notation for the mathematical description of the problem of finding the maximum clique. $G=(V, E)$ is an arbitrary undirected graph, $V=\{1,2, \ldots$, $n\}$ is the set of vertices (nodes) of $G, E \subseteq V \times V$ is the set of edges (arcs) of $G$, and denote $\bar{E}=\{(i, j): i, j \in V, i \neq j,(i, j) \notin E\}$. To find the maximum clique of $G$, we needed to solve the following problem:

$$
\begin{gathered}
\max _{y \in Y} \sum_{i \in V} y_{i}, \\
Y=\left\{y \in\{0,1\}^{n}: y_{i}+y_{j} \leq 1 \forall(i, j) \in \bar{E}\right\} .
\end{gathered}
$$

Let $S O L(G)$ be the set of solutions of Problem (3) for the graph $G$, and let $\Omega=(I, S)$ be the graph that corresponds to the gas transmission system described above. Then every $y \in \operatorname{SOL}(\Omega)$ determines the set of nodes of the gas transmission system that form a clique of maximum size. In the case of non-uniqueness of the solution, we obtained a set of such cliques, which can serve as the basis for further more detailed planning of an attack with maximum damage on the part of the attacker.

\subsubsection{Determination of Gas Pipelines Cliques}

Solving Problem (3) on the original graph $\Omega$ will allow the attacker to determine the cliques associated with the nodes of gas production, consumption, storage, and branch nodes. To detect the clique of gas lines, it was necessary to make the transition from the original graph to the edge one. This can be done as follows.

Let there be an arbitrary directed graph $G$, given by a set of vertices $V$ and edges $E$. An edge graph is obtained from the original one by representing the edges of the original graph as vertices. Let us consider the scheme of transformation of an initial graph into an edge one using a simple example. In Figure 1, on the left, a graph $G$ is given, consisting of five vertices and six directed edges; on the right, its edge graph $L(G)$ is constructed, which is defined as follows:

1. Any vertex of the graph $L(G)$ represents an edge of the graph $G$;

2. Two vertices of the graph $L(G)$ are adjacent if and only if their corresponding edges have a common vertex in $G$.
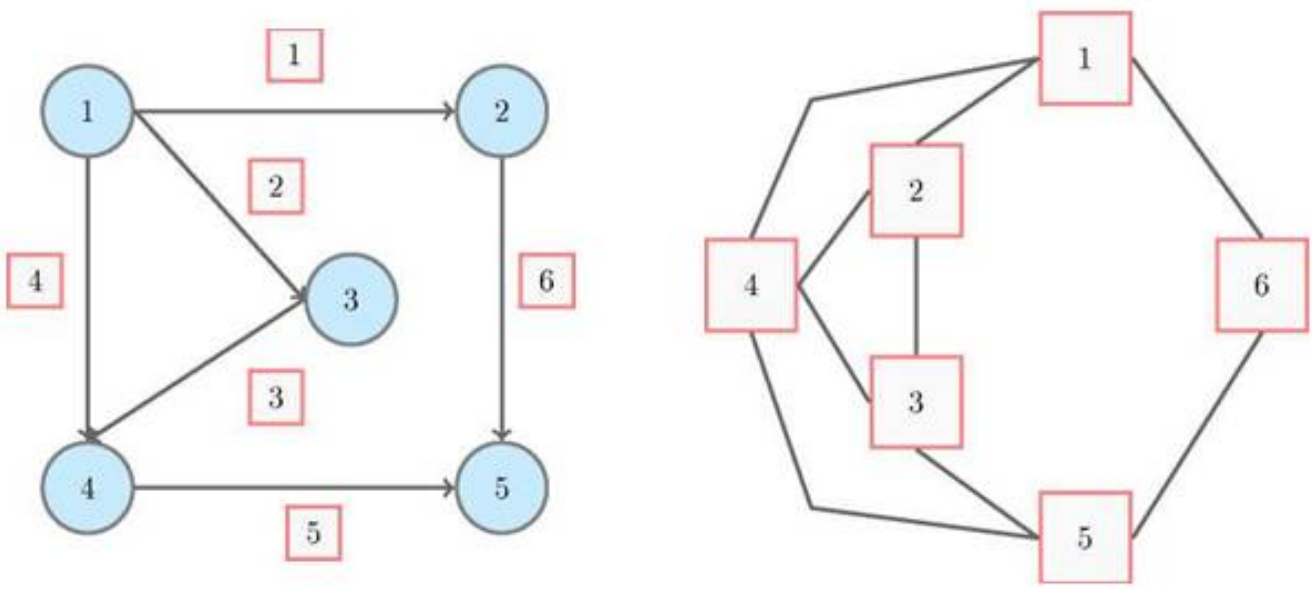

Figure 1. Original graph (left) and edge graph (right).

To form an adjacency matrix of an edge graph, we constructed an incidence matrix $A$ of size $m \times n$ of the original graph. Constants $m$ and $n$ determine the number of rows and 
columns in this matrix and correspond to the number of edges and the number of vertices in the graph. For our example, $m=6$ and $n=5$. The elements $a_{i j}$ of the incidence matrix $A$ took the following values:

$$
a_{i j}=\left\{\begin{array}{c}
1, \text { if the } i \text {-th edge leaves the } j-\text { th node, } \\
0, \text { if the } i-\text { th edge is not connected with the } j \text {-th node, } \\
-1, \text { if the } i \text {-th edge enters the } j-\text { th node. }
\end{array}\right.
$$

Below are the incidence matrix of the original graph (matrix $A$ ) and the adjacency matrix of the edge graph (matrix $B$ ):

$$
A=\left(\begin{array}{rrrrr}
1 & -1 & 0 & 0 & 0 \\
1 & 0 & -1 & 0 & 0 \\
0 & 0 & 1 & -1 & 0 \\
1 & 0 & 0 & -1 & 0 \\
0 & 0 & 0 & 1 & -1 \\
0 & 1 & 0 & 0 & -1
\end{array}\right), B=\left(\begin{array}{llllll}
0 & 1 & 0 & 1 & 0 & 1 \\
1 & 0 & 1 & 1 & 0 & 0 \\
0 & 1 & 0 & 1 & 1 & 0 \\
1 & 1 & 1 & 0 & 1 & 0 \\
0 & 0 & 1 & 1 & 0 & 1 \\
1 & 0 & 0 & 0 & 1 & 0
\end{array}\right) .
$$

Matrix $B$ was constructed from the incidence matrix of the original graph as follows. Take, for example, the first column of the original matrix $A$, which corresponded to the first vertex. From this vertex, there were three edges: 1,2, and 4 (the corresponding elements of the first, second, and fourth rows in the first column were equal to 1 ). The indicated edges were pairwise adjacent to each other through the first node; therefore, in the adjacency matrix $B$ for vertex 1 , we set connections with vertices 2 and 4 , and did not forget to set the connection between the second and fourth vertices. We proceeded in the same way when considering the remaining columns of matrix $A$. Figure 2 presents the cliques of the edge graph (on the right) and the corresponding sections of the original graph (on the left).
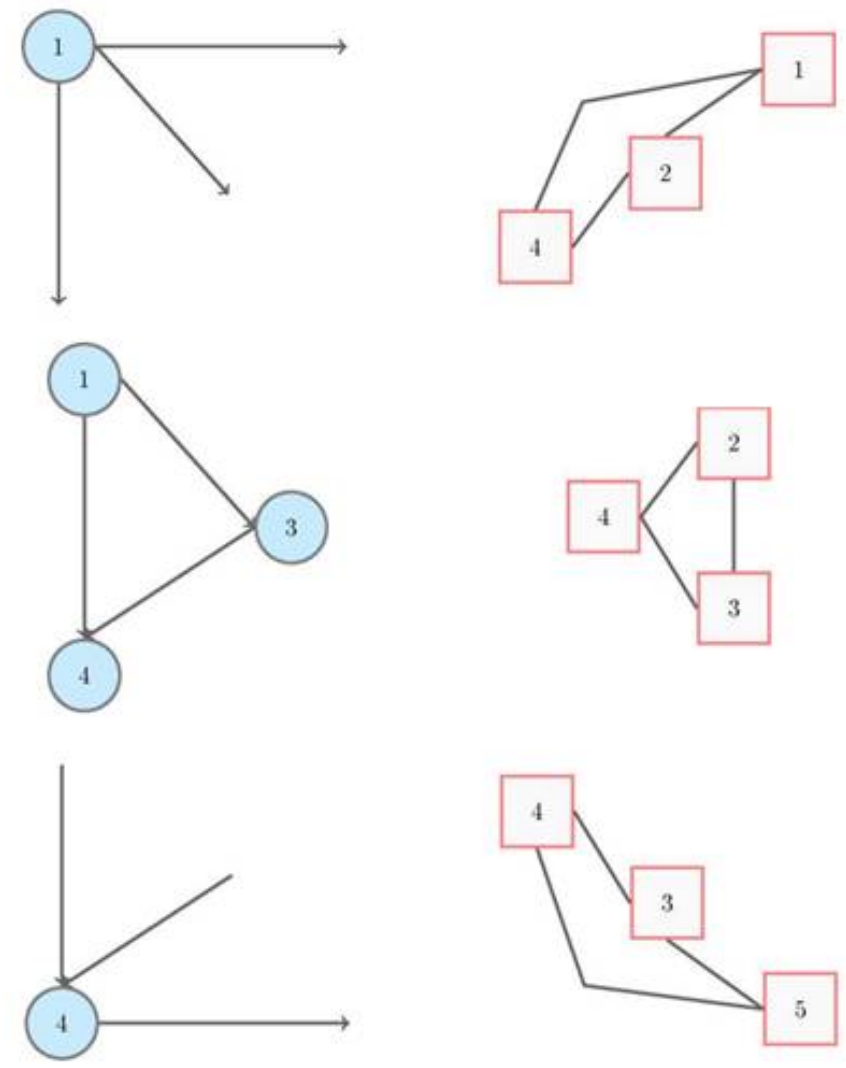

Figure 2. The cliques' correspondence of the edge graph (on the right) to the sections of the original graph (on the left). 
The proposed scheme for transforming the initial graph into an edge graph provides the attacking side with a more extensive set of actions causing maximum damage to the defender. Solving the problem of the maximum clique on the initial graph $\Omega$ allows for planning an attack on such objects of the gas transmission system, such as fields, gas storage reservoirs, and compressor stations. Furthermore, in the case of solving the same problem for an edge graph $L(\Omega)$, the attacker can estimate the damage in the case of failure of the gas pipeline system. Thus, we arrived at an attacker-defender type problem in the following form:

$$
\begin{gathered}
\min _{y} \max _{x} f, \\
y \in \operatorname{SOL}(L(\Omega)), \\
\sum_{j \in I} \bar{u}_{i j} x_{i j}=\left\{\begin{array}{c}
-f, i=i_{O}, \\
0, i \notin\left\{i_{O}, i_{S}\right\}, \quad i \in I, \\
f, i=i_{S},
\end{array}\right. \\
x_{i j}=x_{j i}, i, j \in I \backslash\left\{i_{O}, i_{S}\right\}, \\
0 \leq x_{i j} \leq d_{i j}\left(1-y_{i j}\right), i \in I, j \in I,
\end{gathered}
$$

where $y_{i j}=y_{j i}$ stands for the component of $y$ that corresponds to the arc $(i, j)$. Thus, a maximum clique $y \in \operatorname{SOL}(L(\Omega))$ is a $\{0,1\}$-vector that determines the nodes of the edge graph, namely, the pipelines to which the attack is directed (the attacker's plan). The pipeline $(i, j)$ is in the attacker's plan if $y_{i j}=1$, and is not otherwise.

Based on the solution of Problem (4), the attacker selects such a clique, the removal of which will lead to the greatest gas shortage among consumers. The attacked cliques are removed from the calculation network.

In this study, combinations of sections of gas pipelines, which were determined using the maximum clique method, referred to critical facilities of the gas industry if the gas deficit during their shutdown was $10 \%$ or more in the system. This statement was made in accordance with the previously formulated methodology for determining critical objects of power systems from the standpoint of ensuring the operability of these systems [17] and earlier studies [14].

The model of the Russian gas transmission system was described by means of the algebraic modeling system AIMMS 4.66 [33]. In this system, the problem of the maximum flow (2) and the maximum clique problem (3) were solved using CPLEX 12.9 solver. The calculations were carried out on an eight-core AMD FX-8350 processor PC (clock frequency of each core was $4 \mathrm{GHz}$ ) and $8 \mathrm{~GB}$ of RAM.

\section{Results}

As part of the design conditions in this study, the average day in January was taken, when in the constituent entities of the Russian Federation, there is the maximum consumption of natural gas with its additional delivery from the underground gas storage reservoirs. Thus, these are the most difficult conditions for the functioning of the gas transmission network, under which, it is maximally loaded. In January 2019, the average load on the Russian gas transmission network was 2234.87 million $\mathrm{m}^{3}$ /day.

Figure 3 shows an enlarged design scheme of the Unified Gas Supply System of Russia with access to European countries. Today, more than $90 \%$ of Russian natural gas is produced in one gas-producing region (North of the Tyumen region). This region is located at a distance of 2-2.5 thousand kilometers from the main gas-consuming regions and $4-5$ thousand kilometers from importing countries. Thus, the Russian gas transmission system is an extensive, geographically distributed network of gas pipelines with a large number of crossings and branches. In addition, the corridors of large gas pipelines often run close to each other. 


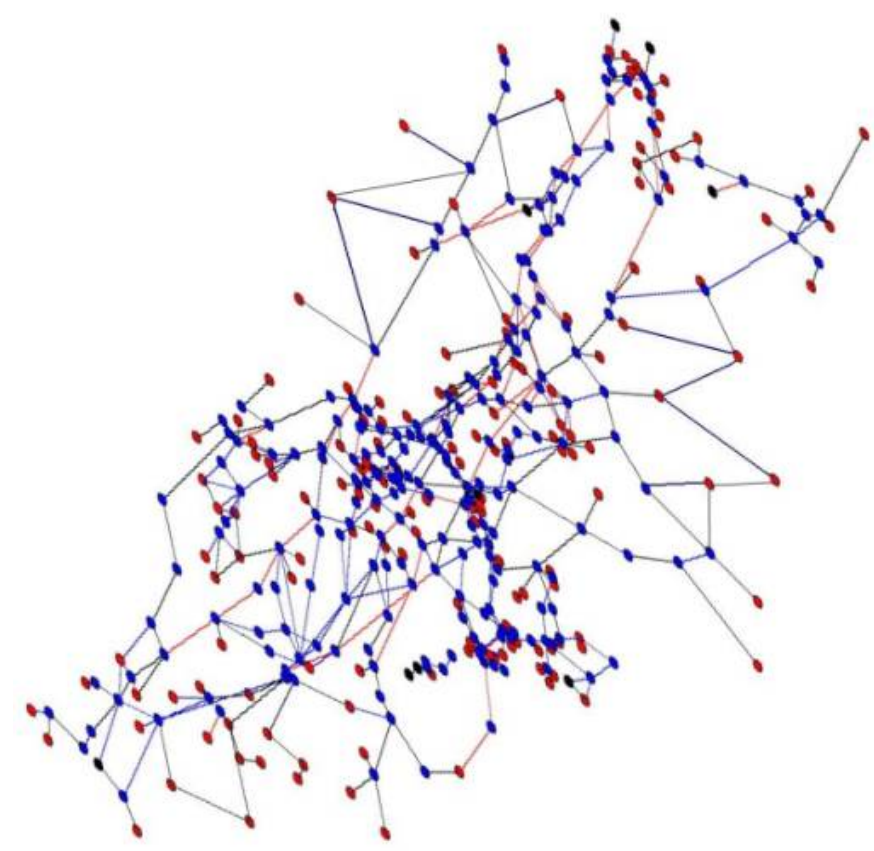

Figure 3. Enlarged calculation scheme of the Unified Gas Supply System of Russia.

As noted earlier, the nodes in an edge graph represent the edges of the original graph. Consequently, the search for a clique of the maximum size in an edge graph can be interpreted as a search for a node or a system of nodes through which the maximum number of gas pipelines is laid. The maximum flow Problem (4) was solved individually for every clique $y \in S O L(L(\Omega))$. With Problem (4) solved, the gas flow aTnd shortage value were recorded for each of the cliques found in Problem (3). Table 1 lists the cliques found and the damage caused by excluding them from the system. Due to the fact that all studies were carried out on a model that was as close as possible to the actual functioning gas transmission network, the calculation results do not provide either the names of the objects that form the cliques, or information about their location.

Table 1. List of received edge graph cliques.

\begin{tabular}{|c|c|c|c|c|}
\hline № & $\begin{array}{l}\text { Excluded } \\
\text { Clique Size }\end{array}$ & $\begin{array}{l}\text { Gas Flow in the } \\
\text { System } \\
\text { (Million } \mathrm{m}^{3} / \text { Day) }\end{array}$ & $\begin{array}{c}\text { Gas Shortage } \\
\left.\text { (Million } \mathrm{m}^{3} / \mathrm{Day}\right)\end{array}$ & Gas Shortage (\%) \\
\hline 1 & 4-edge clique & 1850.9 & 383.9 & 17.2 \\
\hline 2 & 4-edge clique & 1850.9 & 383.9 & 17.2 \\
\hline 3 & 3-edge clique & 1850.9 & 383.9 & 17.2 \\
\hline 4 & 2-edge clique & 1932.4 & 302.4 & 13.5 \\
\hline 5 & 5-edge clique & 2002.2 & 232.6 & 10.4 \\
\hline 6 & 4-edge clique & 2043.0 & 191.8 & 8.6 \\
\hline 7 & 6-edge clique & 2104.0 & 130.8 & 5.9 \\
\hline 8 & 7-edge clique & 2111.6 & 123.2 & 5.5 \\
\hline 9 & 3-edge clique & 2118.3 & 116.5 & 5.2 \\
\hline 10 & 4-edge clique & 2136.6 & 98.2 & 4.4 \\
\hline 11 & 4-edge clique & 2174.3 & 60.5 & 2.7 \\
\hline 12 & 4-edge clique & 2186.9 & 47.9 & 2.1 \\
\hline 13 & 4-edge clique & 2212.0 & 22.8 & 1.0 \\
\hline
\end{tabular}

Table 1 shows data on the maximum flow while accounting for the excluded cliques. Considering that the number of cliques detected was large, Table 1 shows the cliques that caused significant damage to the gas transmission network. Table 1 clearly demonstrates that the failure of the first three cliques of the edge graph will lead to a gas deficit for 
consumers in $17 \%$ for the system as a whole. This gas deficit is comparable to the failure of other critical objects identified in previous studies [14,17].

As a result of the study [17], 61 critically important objects of the gas industry were identified. Gas deficits arising from the failure of these objects varied in the range from $5 \%$ to $21 \%$. Taking the $5 \%$ gas shortage as the lower bound, the nine cliques found in this study met the criteria for critical gas objects. Due to the fact that the found cliques are interconnected combinations of two or more objects, searching for them using the enumeration method, as in the study [17], is very difficult.

The study [14] confirmed the hypothesis that the joint failure of a critical object and any other object in the network can lead to a significantly greater gas shortage among consumers. The enumeration method found 61,035 pairs of objects consisting of a critical object and some other network object. Such failures lead to gas deficits in the range from $5 \%$ to $32 \%$. After analyzing the results, 630 pairs of objects were identified, leading to gas deficits from $10 \%$ to $32 \%$. In the presented study, the results were obtained much faster; a large amount of work was not required to analyze and sort the received cliques. Nevertheless, the cliques found in this study corresponded to the results of [14] in terms of possible gas shortages in the event of failure of the found network objects.

In the study [13], 20 edges of the calculation scheme of the gas transmission network were determined. First, the failure of these edges will lead to a gas shortage of $3-21 \%$. Second, these sections make the greatest contribution to the possible synergistic effect that is possible in the case of the failure of several sections of the network. Such a synergistic effect lies in the fact that joint disruption of the operation of several network objects will create a gas deficit greater than the total deficit if these objects are disconnected separately. The results of this study corresponded to the results obtained in [13] regarding the volumes of gas deficit among consumers when cliques are turned off. The found cliques were not analyzed for the presence of synergistic effects because, in this formulation of the problem, situations were simulated with the failure of entire network segments that form cliques.

In the study [34], combinations of interconnected nodal compressor stations that form cliques were determined. A total of 45 cliques were found consisting of three elements. Of the received cliques, six were selected, the failure of which will lead to a gas deficit in the amount of $11-39 \%$. It should be noted that some of the cliques contain critical objects found earlier in [17]. The results of this study were consistent with the results of [34], both in terms of possible gas deficits and in terms of the dimensions of the resulting cliques. Nevertheless, the importance of the results obtained lies in the fact that they confirmed the possibility of applying a new approach to finding critical network objects. This approach consisted in analyzing the structure of the throughput capacity of interconnected gas pipelines, and not simply in enumerating combinations of nodal compressor stations.

In total, with this approach to the analysis of the edge graph, 13 cliques were identified, the exclusion of which would lead to a gas shortage among consumers. For the first five cliques, the failure of these can lead to a gas shortage of $10-17 \%$ for the system as a whole; therefore, they can be considered critical objects in the gas industry.

The difference in gas shortages among consumers in the event of failure of various cliques was due to several factors. First of all, this was the different throughput capacities of the gas pipelines that formed the resulting cliques. Basically, these were several gas pipelines with a diameter of $1420 \mathrm{~mm}$. In cases with six- and seven-edge cliques, these were gas pipelines of smaller diameters. The locations of the found cliques and, accordingly, the ability of the gas transmission system to bypass the excluded objects also affected the gas deficit among consumers.

\section{Discussion and Conclusions}

The identification of critical combinations of objects in the gas transmission network allows for planning protective measures and reducing potential gas shortages among consumers in the event of an attack. The article proposed the application of the method for determining the maximum cliques to search for the most interconnected sections of 
main gas pipelines of a gas transmission network. Solving the problem for the maximum clique helps to assess the significance of a particular combination of network objects from the point of view of providing consumers with gas. At the same time, one can proceed to the analysis of larger subsystems of nodes without using iterative procedures for the step-by-step elimination of network elements and their combinations, which complements the previous work done on this topic. The study was carried out on the calculation scheme of the Unified Gas Supply System of Russia. The most interconnected sections of the network were identified and the analysis of possible consequences for the system in case of their shutdown was carried out.

First, it should be noted that the maximum size of the edge clique for the Russian gas transmission network was seven interconnected elements. In contrast, the maximum node clique in this network was three elements [34].

Second, as a result of the application of the proposed method, five interconnected sections of main gas pipelines were obtained, which formed cliques, the failure of which can lead to a gas deficit in the amount of $10-17 \%$ for the system as a whole. Based on the high importance of these cliques in providing consumers with gas, the authors of this article proposed to include them in the list of critical objects of the gas industry.

Third, gas deficits arising from the failure of the found cliques had deficits resulting from the failure of critical objects of the gas industry in the range of $5-21 \%$ and $10-32 \%$ for their combinations [14,17].

The calculation results corresponded to the results of previous studies performed using other methods, such as element-by-element enumeration [14,17], the method for determining synergistic effects [13], and the clique method for network nodes [34]. Thus, the presented study and the results of calculations confirmed the applicability of the method of maximum cliques for identifying critical objects in the gas industry. This method can be used when searching for critical elements in critical network infrastructures.

As a further development of research, the authors see the development of measures aimed at reducing the significance of the found cliques. Such events should be both shortterm and long-term. Thus, taking such measures into account in the further development of the gas transmission system will help to increase its reliability.

Author Contributions: Conceptualization, S.V. and A.K.; methodology, S.V. and A.K.; software, A.K. and I.M.; validation, A.K. and I.M.; formal analysis, S.V. and A.K.; investigation, A.K.; resources, I.M.; data curation, I.M.; writing—original draft preparation, S.V. and A.K.; writing—review and editing, I.M.; supervision, S.V.; project administration, S.V.; funding acquisition, S.V. All authors have read and agreed to the published version of the manuscript.

Funding: This research was funded by the Russian Science Foundation, grant no. 20-79-00242, using the resources of the High-Temperature Circuit Multi-Access Research Center (Ministry of Science and Higher Education of the Russian Federation, project no. 13.CKP.21.0038).

Institutional Review Board Statement: Not applicable.

Informed Consent Statement: Not applicable.

Data Availability Statement: Not applicable.

Conflicts of Interest: The authors declare no conflict of interest. The funders had no role in the design of the study; in the collection, analyses, or interpretation of data; in the writing of the manuscript; or in the decision to publish the results.

\section{References}

1. Accidents on Main Gas Pipelines in Russia in 2018-2019. Available online: ria.ru/20190728/1556953028.html (accessed on 6 December 2021).

2. Top 5 Largest and Most Devastating Gas Pipeline Accidents. Available online: sila-sibiri-rabota.ru/avarii-na-gazoprovodax/ (accessed on 6 December 2021).

3. Wang, W.C.; Zhang, Y.; Li, Y.X.; Hu, Q.; Liu, C.; Liu, C. Vulnerability analysis method based on risk assessment for gas transmission capabilities of natural gas pipeline networks. Reliab. Eng. Syst. Saf. 2022, 218 Pt B, 108150. [CrossRef] 
4. Yu, W.; Huang, W.; Wen, Y.; Li, Y.; Liu, H.; Wen, K.; Gong, J.; Lu, Y. An integrated gas supply reliability evaluation method of the large-scale and complex natural gas pipeline network based on demand-side analysis. Reliab. Eng. Syst. Saf. 2021, $212,107651$. [CrossRef]

5. Tichý, L. Energy Infrastructure as a Target of Terrorist Attacks from the Islamic State in Iraq and Syria. Int. J. Crit. Infrastruct. Prot. 2019, 25, 1-13. [CrossRef]

6. Tsavdaroglou, M.; Al-Jibouri, S.H.S.; Bles, T.; Halman, J.I.M. Proposed Methodology for Risk Analysis of Interdependent Critical Infrastructures to Extreme Weather Events. Int. J. Crit. Infrastruct. Prot. 2018, 21, 57-71. [CrossRef]

7. Han, F.; Zio, E. A Multi-Perspective Framework of Analysis of Critical Infrastructures with Respect to Supply Service, Controllability and Topology. Int. J. Crit. Infrastruct. Prot. 2019, 24, 1-13. [CrossRef]

8. Ouyang, M. A Mathematical Framework to Optimize Resilience of Interdependent Critical Infrastructure Systems under Spatially Localized Attacks. Eur. J. Oper. Res. 2017, 262, 1072-1084. [CrossRef]

9. Emenike Scholastica, N.; Falcone, G. A Review on Energy Supply Chain Resilience through Optimization. Renew. Sustain. Energy Rev. 2020, 134, 110088. [CrossRef]

10. Aljaroudi, A.; Khan, F.; Akinturk, A.; Haddara, M.; Thodi, P. Risk Assessment of Offshore Crude Oil Pipeline Failure. J. Loss Prev. Process Ind. 2015, 37, 101-109. [CrossRef]

11. Chen, C.; Li, C.; Reniers, G.; Yang, F. Safety and Security of Oil and Gas Pipeline Transportation: A Systematic Analysis of Research Trends and Future Needs Using WoS. J. Clean. Prod. 2021, 279, 123583. [CrossRef]

12. Senderov, S.M.; Edelev, A.V. Formation of a List of Critical Facilities in the Gas Transportation System of Russia in Terms of Energy Security. Energy 2019, 184, 105-112. [CrossRef]

13. Vorobev, S.; Edelev, A. Analysis of the importance of critical objects of the gas industry with the method of determining critical elements in networks of technical infrastructures. In Proceedings of the 2017 Tenth International Conference Management of Large-Scale System Development (MLSD), Moscow, Russia, 2-4 October 2017; IEEE: Piscataway, NJ, USA, 2017 ; pp. 1-4. [CrossRef]

14. Vorobev, S.; Edelev, A.; Smirnova, E. Search of critically important objects of the gas industry with the method of determining critical elements in networks of technical infrastructures. In Proceedings of the Methodological Problems in Reliability Study of Large Energy Systems (RSES 2017), Bishkek, Kyrgyzstan, 11-15 September 2017; Volume 25. [CrossRef]

15. Senderov, S.; Krupenev, D. Energy Security and Critical Facilities of Energy Systems: Methodology and Practice of their Identification on the Example of Russia's Gas and Electric Power Industries. Energy Syst. Res. 2019, 2, 41-50.

16. Senderov, S.M.; Smirnova, E.M.; Vorobev, S.V. Analysis of Vulnerability of Fuel Supply Systems in Gas-Consuming Regions Due to Failure of Critical Gas Industry Facilities. Energy 2020, 212, 118785. [CrossRef]

17. Senderov, S.M.; Vorobev, S.V. Approaches to the Identification of Critical Facilities and Critical Combinations of Facilities in the Gas Industry in Terms of Its Operability. Reliab. Eng. Syst. Saf. 2020, 203, 107046. [CrossRef]

18. Von Stackelberg, H.; Peacock, T. The Theory of the Market Economy. Economica 1953, 20, 384. [CrossRef]

19. Alderson, D.L.; Brown, G.G.; Carlyle, W.M.; Wood, R.K. Solving Defender-Attacker-Defender Models for Infrastructure Defense. In Proceedings of the 12th INFORMS Computing Society Conference, Presented at the Operations Resaerch, Computing, and Homeland Defense, INFORMS, Monterey, CA, USA, 9-11 January 2011; pp. 28-49. [CrossRef]

20. Brown, G.; Carlyle, M.; Salmerón, J.; Wood, K. Defending Critical Infrastructure. Interfaces 2006, 36, 530-544. [CrossRef]

21. Cole Smith, J.; Lim, C. Algorithms for Network Interdiction and Fortification Games. In Pareto Optimality, Game Theory and Equilibria; Chinchuluun, A., Pardalos, P.M., Migdalas, A., Pitsoulis, L., Eds.; Springer Optimization and Its Applications; Springer: New York, NY, USA, 2008; pp. 609-644. [CrossRef]

22. Manshadi, S.D.; Khodayar, M.E. Resilient Operation of Multiple Energy Carrier Microgrids. IEEE Trans. Smart Grid 2015, 6, 2283-2292. [CrossRef]

23. Wang, C.; Wei, W.; Wang, J.; Liu, F.; Qiu, F.; Correa-Posada, C.M.; Mei, S. Robust Defense Strategy for Gas-Electric Systems Against Malicious Attacks. IEEE Trans. Power Syst. 2017, 32, 2953-2965. [CrossRef]

24. Wu, X.; Conejo, A.J. An Efficient Tri-Level Optimization Model for Electric Grid Defense Planning. IEEE Trans. Power Syst. 2017, 32, 2984-2994. [CrossRef]

25. Collins, M.; Cooper, L.; Helgason, R.; Kennington, J.; LeBlanc, L. Solving the pipe network analysis problem using optimization techniques. Manag. Sci. 1978, 24,747-760. [CrossRef]

26. Beckmann, M.J. On the theory of traffic flows in networks. Traffic Q. 1967, 21, 109-116.

27. Israeli, E.; Wood, R.K. Shortest-Path Network Interdiction. Networks 2002, 40, 97-111. [CrossRef]

28. Exports of the Russian Federation of the Most Important Goods in 2015-2019 (According to the Federal Customs Service of Russia). Available online: customs.ru/index.php?option=com_newsfts\&view=category\&id=52\&Itemid=1978\&limitstart $=60$ (accessed on 6 December 2021).

29. Energy production summary. InfoTEK Mon. Oil Gas Mag. 2020, 1, 113-131. (In Russian)

30. Ministry of Energy of the Russian Federation. Statistics. Available online: minenergo.gov.ru/activity/statistic (accessed on 6 December 2021).

31. Korotaev, Y.; Margulov, R. Extraction, Preparation and Transportation of Natural Gas and Condensate; Nedra: Moscow, Russia, 1984; Volume 2. (In Russian)

32. Du, D.-Z.; Pardalos, P.M. (Eds.) Handbook of Combinatorial Optimization; Springer: New York, NY, USA, 1999. [CrossRef] 
33. AIMMS Homepage. Available online: aimms.com (accessed on 6 December 2021).

34. Vorobev, S.; Kolosnitsyn, A.; Minarchenko, I. The clique approach to identifying critical elements in gas transmission networks. In Proceedings of the International Conference of Young Scientists “Energy Systems Research 2021", Irkutsk, Russia, 25-28 May 2021; Volume 289. [CrossRef] 